\title{
OPTICAL OBSERVATIONS OF SMALL SPACECRAFT AND SPACE DEBRIS AT ISTP SB RAS SAYAN OBSERVATORY
}

\author{
I.V. Korobtsev \\ Institute of Solar-Terrestrial Physics SB RAS, \\ Irkutsk, Russia, korobtsev@yandex.ru
}

\author{
M.N. Mishina \\ Institute of Solar-Terrestrial Physics SB RAS, \\ Irkutsk, Russia,mmish@iszf.irk.ru
}

\begin{abstract}
Launch of several thousands of small spacecraft as part of satellite groups of various companies (OneWeb, SpaceX, etc.) is expected in the coming years. They will significantly increase the "population" in the low-orbit region. The spacecraft are designed mainly to provide telecommunications and remote sensing of Earth from space. They have a short lifetime. The use of high-speed sCMOS (scientific complementary metal-oxide-semiconductor) detectors in combination with medium diameter optical telescopes provides high temporal resolution, which allows us to get detailed brightness light curves and to estimate the state of small spacecraft. We present the technique and results of photometric measurements of small spacecraft obtained
\end{abstract}

with the 1.6-meter AZT-33IK telescope of ISTP SB RAS Sayan Solar Observatory. Photometric measurements are shown to be an important source of information at various operational stages of satellites.

Keywords: small spacecraft, low-Earth orbit, photometry, optical telescope.

\section{INTRODUCTION}

One of the main directions for the development of space industry emerging in recent years is the design, development, and application of small spacecraft (SS). The shortened timeline of developing SS and the relatively low cost of orbital injection allow their effective use for a wide range of tasks - research, educational, social and economic, and defense. In the XXI century, the development of CubeSat satellites [Woellert et al., 2011] by the standard established by California Polytechnic State University and Stanford University has become a widespread phenomenon. The satellites have a size of $10 \mathrm{~cm} \times 10 \mathrm{~cm} \times 10 \mathrm{~cm}$ and a weight of no more than $1.33 \mathrm{~kg}$. The standard allows the combination of two or three standard cubes in one satellite (denoted by $2 \mathrm{U}$ and $3 \mathrm{U}$ and have a size of $10 \mathrm{~cm} \times 10 \mathrm{~cm} \times 20 \mathrm{~cm}$ or $10 \mathrm{~cm} \times 10 \mathrm{~cm} \times 30 \mathrm{~cm}$ ). According to SpaceWorks company data [Nano/Microsatellite Forecast, 2019], the expected number of nanosatellites and microsatellites will continue to increase and in the next five years will exceed 2000.

Most small spacecraft are launched into low Earth orbits with a height from 700 to $3000 \mathrm{~km}$, their observation has its own specifics and places particular requirements on the equipment, observation scheduling, and measurement processing. In high orbits there are also SS, and their number will only increase in the coming years. Launch of a large number of SS is often accompanied by disruptions, breakdown of stabilization, and absence of telemetry information from SS.

Incidents, technical anomaly or divergence of technical parameters from the norm result in changes of reflection characteristics of SS surfaces or the nature of its motion, which can be detected by ground-based astronomical instruments. The analysis of the reflection characteristics of SS obtained from photometric observations made by optical telescopes provide information about spacecraft shape, its orientation and stabilization. The set of photometric data (light curves, phase curves, spectral characteristics) enables us to make assumptions about spacecraft (SC) shape and dimensions; if it rotates, to determine its speed and orientation of axes, to study reflective properties of SC and their changes over time. These photometric observations can detect such malfunctions in SC as stabilization system breakdown, change of spatial orientation, absence of some details in the SC structure. For SC in orbits above $3000-5000 \mathrm{~km}$, the photometric information is virtually the only available for SC monitoring and evaluation of their technical state.

In this paper, we report the results of photometric observations of small spacecraft, obtained with the 1.6meter telescope AZT-33IK at ISTP SB RAS Sayan Solar Observatory. We have demonstrated the effectiveness of telescopes with high limiting magnitude in studying optical characteristics of small objects, obtaining light curves with high temporal resolution and simultaneously with high accuracy.

\section{SPECIAL PURPOSE TELESCOPE AZT-33IK}

The telescope AZT-33IK of ISTP SB RAS Sayan Solar Observatory can make trajectory and photometric measurements of artificial earth satellites and space debris in the optical and IR ranges in orbits of all types. The optical scheme of the telescope is designed in analogy with the Ritchey-Chrétien scheme with an equivalent focal length of $30 \mathrm{~m}$; the telescope aperture is 1.6 $\mathrm{m}$, the angular diameter of its field of view is $10 \mathrm{arcmin}$ [Kamus et al., 2002]. The plane of the Cassegrain focus of the telescope is at a distance of $1015 \mathrm{~mm}$ from the 
top of the primary mirror, which allowed us to install a diagonal mirror in front of it, which can divert a beam to various devices located behind the primary mirror barrel. Currently, photometric measurements of SC and space debris are being made using a photometer consisting of a field-of-view reducer, a turret of Johnson filters BVRI [Bessell, 2005], and an ANDOR Neo 5.5 sCMOS camera with a $2560 \times 2160$ array $6.5 \mu \mathrm{m} \times 6.5 \mu \mathrm{m}$ in size (corresponding to the field of view of $8.3 \times 7 \mathrm{arcmin}$ ).

\section{FEATURES OF SPACECRAFT MEASUREMENTS IN LOW-EARTH ORBITS}

Optical observations of LEO SC (LSC) have their own specific features and set requirements for equipment such as high-precision object tracking according to the ephemeris in a wide range of angular velocities (up to several degrees per second), a wide dynamic range of photodetector, possibility of obtaining short exposures (a fraction of a second), and fast frame readout.

Typically, due to high LSC velocities, the star tracks on the frames taken with the main optical system of the AZT-33IK telescope having a field of view of $8 \times 7$ arcmin are too long, even at short exposures (shorter than $1 \mathrm{~s}$ ), as shown in Figure 1.

The length of the tracks do not allow us to match stars visible in the frame to the star catalog, as is done during SC observations in high and medium-earth orbits. A necessary stage of LSC observations is, therefore, to obtain calibration frames with star fields. During LSC flight, the zenith distance may vary over a wide range, and hence the sunlight reflected from the spacecraft passes through absorbing atmospheric layers with different optical thicknesses. The star fields are measured along the entire trajectory of the object, which allows us to account for atmospheric extinction. In these fields, we select stars with known magnitudes. The extinction coefficient is determined by solving a redundant system of equations [Mironov, 2008]

$$
M_{i}=m_{i}-k X_{i}+N P,
$$

where $M_{i}$ is the standard magnitude of the $i$ th star, $m_{i}$ is the instrumental magnitude of the $i$ th star, $k$ is the extinction coefficient, $X_{i}$ is the air mass of the $i$ th star, NP is the zero point of the instrumental system.

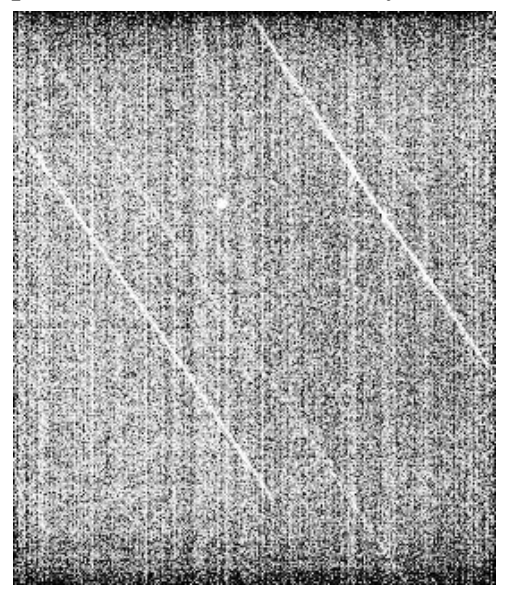

Figure 1. Frame obtained from LSC measurement using the AZT-33IK main optical system with the $0.5 \mathrm{~s}$ exposure

A further step required for the correct interpretation and comparison of LSC light curves is a procedure for reducing the LSC brightness to one distance [Kulikovskiy, 2002]. For an object located at a distance $R$ from Earth, the magnitude $M_{\mathrm{o}}$, reduced to the distance $R_{\mathrm{o}}$, is calculated from the formula

$$
M_{0}=M-5 \log _{10}\left(R / R_{0}\right) \text {. }
$$

\section{LSC MEASUREMENTS IN LOW-EARTH ORBITS}

For the observations, we have selected both Russian and foreign micro-, nano-, and pico-objects, orbital data on which is available in the open catalog of space objects [https://www.space-track.org], supported by the North American Aerospace Defense (NORAD). Duration of the obtained series of observations determined by the visibility conditions of the satellite from the observatory and was from 2 to $10 \mathrm{~min}$. The exposure time in use was $0.01-1 \mathrm{~s}$.

\section{TabletSat-Aurora}

The first Russian commercial microsatellite (international designation NORAD-2014-033H/40017) is a technological demonstrator designed for testing onboard equipment. The satellite weight is $26 \mathrm{~kg}$, its approximate size is $45 \mathrm{~cm} \times 45 \mathrm{~cm} \times 48 \mathrm{~cm}$. Figure 2 plots satellite brightness, reduced to a distance of $1000 \mathrm{~km}$, as a function of phase angle. The duration of the observation series was $\sim 4$ min (between the motion of SC over the horizon and its entry into the shadow).

The brightness as a function of phase angle has a form typical of the satellites functioning in a normal operation mode. When the phase angle increases to $90^{\circ}$, the satellite brightness decreases, and then starts to increase. The effect of increasing brightness at the phase angles wider than $90^{\circ}$ have been observed previously in GEO and MEO objects [Korobtsev, Mishina, 2017].

\section{Nanosatellite ESTCube-1}

ESTCube-1 (2013-021C/39161) is an Estonian nanosatellite, devised as part of the student space program on the CubeSat technology. The satellite weight is $1.05 \mathrm{~kg}$,

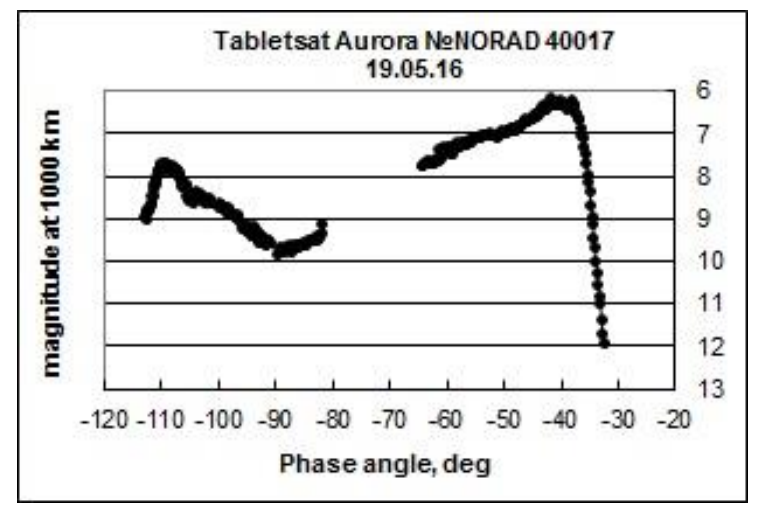

Figure 2. TabletSat-Aurora microsatellite brightness, reduced to a distance of $1000 \mathrm{~km}$, as a function of phase angle 
its size is $10 \mathrm{~cm} \times 10 \mathrm{~cm} \times 11.35 \mathrm{~cm}$. The satellite stopped working, which is confirmed by the obtained light curves suggesting its destabilization and transition to the rotation mode. The behavior of the satellite brightness is rather complicated and does not allow us to determine the proper rotation period.

Figure 3 shows ESTCube-1 brightness values, reduced to a distance of $1000 \mathrm{~km}$, as a function of phase angle for four observation dates.

\section{Picosatellite ITUpSAT-1}

ITUpSAT-1 (2009-051E/35935) is a picosatellite developed at Istanbul Technical University. It is the smallest of the satellites we observed. Its weight is only $0.99 \mathrm{~kg}$, size is $10 \mathrm{~cm} \times 10 \mathrm{~cm} \times 10 \mathrm{~cm}$. The main objective is to study the passive stabilization system, i.e. stabilization by proper rotation, as applied to small spacecraft. With such a stabilization system, SC behaves like a gyroscope, keeping the rotation axis direction unaltered for a long time. The derived light curves show a steady satellite rotation with a period of $9 \mathrm{~s}$ and an amplitude of 1 mag., which confirms the steady operation of the stabilization system. The light curve of the picosatellite derived in the phase angle range of $60^{\circ}$ to $93^{\circ}$ is given in Figure 4.

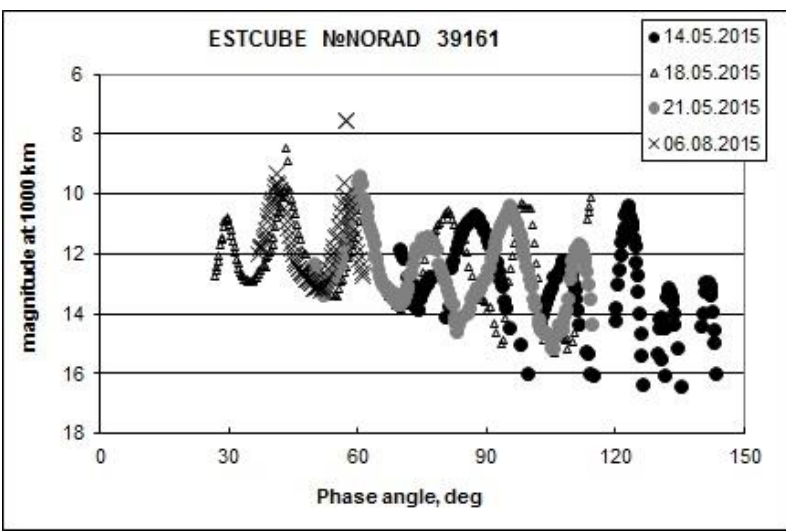

Figure 3. ESTCube-1 nanosatellite brightness, reduced to a distance of $1000 \mathrm{~km}$, as a function of phase angle for four observation dates

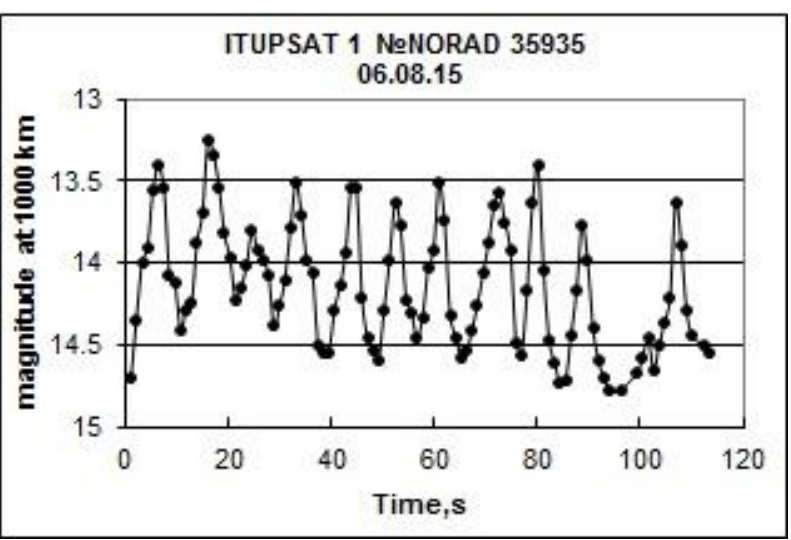

Figure 4. Light curve of the ITUpSAT-1 picosatellite, stabilized by rotation

\section{Small spacecraft from 2017- 042 launch}

In July 2017, the Soyuz-2.1a launch vehicle with Canopus-B-IR SC (international number 2017-042A) was launched from the Baikonur Cosmodrome. As an auxiliary payload there were 72 small satellites of different formats on board Soyuz-2.1a. Shortly after the launch, it was reported that several satellites did not contact or were in an unplanned orbit. Nine objects of this launch, according to the NORAD catalog, remain unidentified to date.

The photometric information about 20 launch objects was obtained using the AZT-33IK telescope. Six of them are unidentified. All of them reveal a variable brightness, which suggests destabilization and rotation or tumbling of the satellite. A light curve of an unidentified satellite rotating with a period of $14.5 \mathrm{~s}$ is presented in Figure 5.

By comparison, Figure 6 shows a light curve of a nominally functioning LSC from this launch, recorded on track between motion of LSC over the horizon and its entry into the shadow.

\section{DISCUSSION}

Let us compare the observation data with theoretical estimates of the LSC brightness. To estime the brightness, we use the formula given in [McCue et al., 1971]:

$$
m=-26.58-2.5 \log _{10}\left(A \gamma F(\varphi) / R^{2}\right),
$$

where $A$ is the area of cross-section of an object, $\gamma$ is the

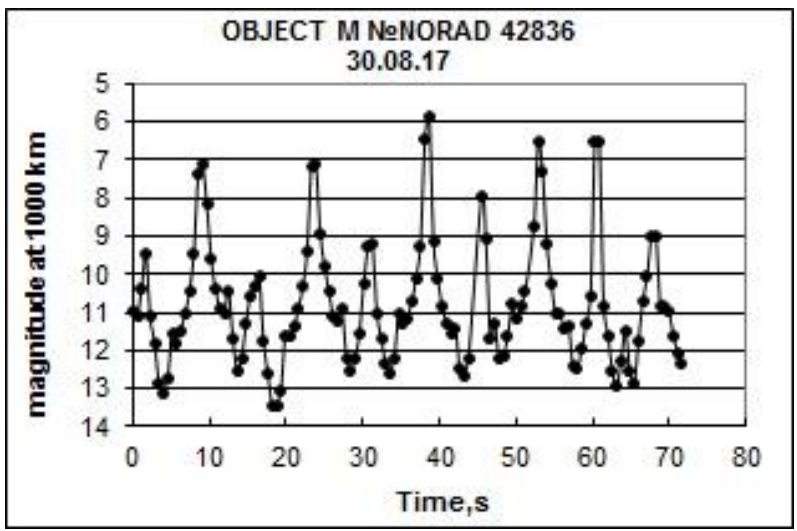

Figure 5. Light curve of LSC with faulty stabilization system from launch 2017-042

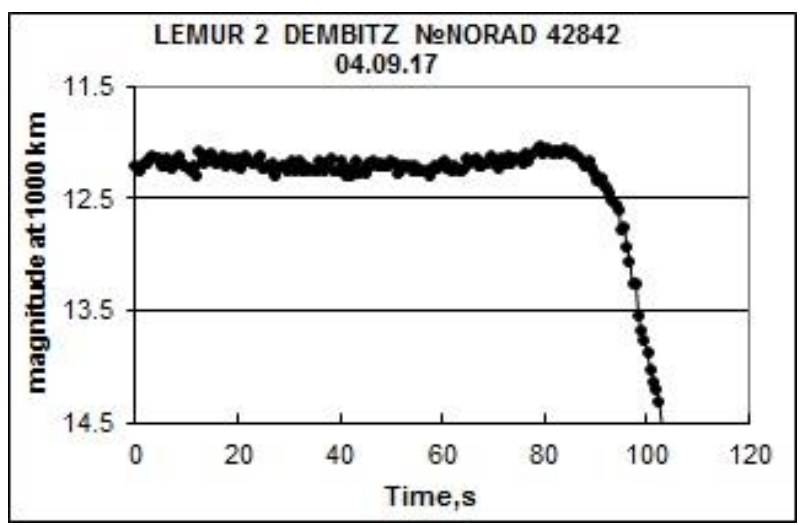
042 
reflection coefficient, $R$ is the distance to the object, $F$ $(\varphi)$ is the phase function.

As a reference object to estimate the brightness, we usually take a sphere with a uniform surface whose phase functions are given by

$$
F(\varphi)=\frac{1}{4 \pi}
$$

for specular reflection,

$$
F(\varphi)=\frac{2}{3 \pi^{2}}[(\pi-\varphi) \cos (\varphi)+\sin (\varphi)]
$$

for diffuse reflection.

Stellar magnitudes for the specular and diffuse spheres of different radii $r$ at a distance $R=1000 \mathrm{~km}$ at a phase angle of $0^{\circ}$ and with a reflection coefficient $\gamma=0.25$ are given in Table.

Estimated apparent brightness for specular and diffuse spheres of different radii

\begin{tabular}{|r|c|c|}
\hline$R, \mathrm{~cm}$ & $m$ (diff.) & $m$ (spec.) \\
\hline 5 & 11.9 & 12.9 \\
\hline 10 & 10.4 & 11.4 \\
\hline 25 & 8.4 & 9.4 \\
\hline
\end{tabular}

Despite the simplified model used to estimate the brightness, the observed brightness of some satellites agrees well with theoretical estimates for the diffuse sphere. Thus, the brightness of the ESTCube- 1 nanosatellite, whose size is $10 \mathrm{~cm} \times 10 \mathrm{~cm} \times 11.35 \mathrm{~cm}$, reduced to a distance of $1000 \mathrm{~km}$, is $\sim 11$ mag. near the zero phase angle.

As seen from the above calculations, the apparent LSC brightness may be great enough due to their close distance to the observer. This is confirmed by observations too. LEO objects whose size exceeds $10 \mathrm{~cm}$ are available for telescopes with a limiting magnitude 10 12 mag. For such objects, the use of wide-aperture telescopes in most cases would be ineffective. The observation of CubeSat objects requires, however, increasing the limiting magnitude of the telescope. The mean brightness of the observed objects of this class at large phase angles is as great as $14-15 \mathrm{mag}$. An even higher limiting magnitude is required for detecting incidents during which there may be wide range changes of brightness, or short-term changes, identification of which requires exposures of a fraction of a second.

\section{CONCLUSIONS}

Using observations of deactivated and active SC as an example, we have demonstrated capabilities of the AZT-33IK telescope to study photometric characteristics of small objects. The telescope with high limiting magnitude, equipped with a photodetector with a high frame readout rate, can identify and analyze incidents when due to vehicle rotation its brightness can vary widely. The mean brightness of the observed objects reaches 14-15 mag.; the minimum brightness, 17 mag.

The analysis of shapes of light curves allows us to find a number of characteristics specific to a particular class of spacecraft, which in some cases can serve as an additional identification feature or provide information about the current state of the spacecraft.

The work was performed with budgetary funding of Basic Research program II.16. The optical observations were made with budgetary funding of major project No. 22 in priority areas determined by the RAS Presidium. The results were obtained using the equipment of Center for Common Use «Angara» [http://ckp-rf.ru/ckp/3056/].

\section{REFERENCES}

Bessell M.S. Standard photometric systems. Ann. Rev. Astron. Astrophys. 2005, vol. 43, pp. 293-336. DOI: 10.1146/annurev.astro.41.082801.100251.

Kamus S.F., Tergoev V.I., Papushev P.G., Druzhinin S.A., Karavaev Y.S., Palachev Y.M., Denisenko S.A., Lipin N.A. Wide range astronomical telescope. Opticheskii zhurnal [J. of Optical Technology]. 2002, vol. 69, no. 9, pp. 84-87. (In Russian).

Korobtsev I.V., Mishina M.N. Investigation of photometrical parameter features of space objects over a wide range of phase angles. Ekologicheskii vestnik nauchnykh tsentrov ChES [Ecological bull. of research centers of the Black Sea Economic Cooperation]. 2017, vol. 4, iss. 2, pp. 60-66. (In Russian). DOI: $10.31429 /$ vestnik-14-4-2-60-66.

Kulikovskiy P.G. Spravochnik lyubitelya astronomii. Izd. 5. [Handbook of amateur astronomy. 5th edition]. Moscow, Editorial URSS Publ., 2002. 688 p. (In Russian).

McCue G.A., Williams J.G., Morford J.M. Optical characteristics of artificial satellite. Planetary and Space Sci. 1971, vol. 19, no. 8, pp. 851-868. DOI: 10.1016/00320633(71)90137-1.

Mironov A.V. Osnovy astrofotometrii. Prakticheskie osnovy fotometrii i spektrofotometrii zvezd [Basics of Astrophotometry. Practical Fundamentals of Photometry and Spectrophotometry of Stars]. Moscow, Fizmatlit Publ., 2008. 260 p.

Nano/Microsatellite market forecast, 9th Edition (2019). Available at: https://www.spaceworks.aero/wp-content/ uploads/Nano-Microsatellite-Market-Forecast-9th-Edition2019.pdf (accessed April 22, 2019).

Woellert K., Ehrenfreund P., Ricco A.J., Hertzfeld H. Cubesats: cost-effective science and technology platforms for emerging and developing nations. Adv. Space Res. 2011, vol. 47, no. 4, p. 663-684. DOI: 10.1016/j.asr.2010.10.009. 2019).

URL: https://www.space-track.org (accessed April 22,

How to cite this article

Korobtsev I.V., Mishina M.N. Optical observations of small spacecraft and space debris at ISTP SB RAS Sayan Observatory. SolarTerrestrial Physics. 2019. Vol. 5. Iss. 4. P. 97-100. DOI: 10.12737/stp54201913. 